\title{
ANÁLISE DA FUSÃO DO PCM ÁCIDO LÁURICO EM CAVIDADE RETANGULAR COM ALETA HORIZONTAL
}

\author{
Aline Morais da Silveira ${ }^{1}$, Iago Dal-Ri ${ }^{1}$ \& Rejane de Césaro Oliveski ${ }^{2}$
}

\begin{abstract}
RESUMO
SILVEIRA, A.M.; DAL-RI, I.; OLIVESKI, R.C. Análise da fusão do PCM ácido láurico em cavidade retangular com aleta horizontal. Perspectivas Online: Exatas \& Engenharias, v. 8, n.20, p.43-55, 2018.
\end{abstract}

Armazenamento de energia térmica é fundamental, seja para climatização, conservação de alimentos, sistemas de refrigeração, entre outras aplicações. Este trabalho apresenta uma análise numérica por CFD (Computational Fluid Dynamics) da fusão do PCM (Phase Change Material) ácido láurico puro em uma cavidade retangular com uma aleta parcial horizontal. O modelo numérico é composto pelas equações de conservação da massa, quantidade de movimento, energia e de mudança de fase. $\mathrm{O}$ estudo consiste em validar a metodologia empregada comparando resultados numéricos com resultados experimentais obtidos da literatura. Como condição de contorno utilizou-se não deslizamento em todas as paredes da cavidade, e para o problema térmico foi utilizada temperatura prescrita na parede vertical direita e na aleta. $O$ comportamento térmico e fluidodinâmico do ácido láurico durante a fusão foi avaliado, visando sua aplicação como PCM. São apresentados campos de temperatura, velocidade e evolução da fração líquida ao longo do tempo, evidenciando que o parâmetro $C$ que melhor representa a fusão do ácido láurico é da ordem de $10^{11}$, e que a convecção natural é o mecanismo mais influente durante o processo fusão.

Palavras-chave: PCM; Ácido láurico; CFD; Aletas. 


\begin{abstract}
Thermal energy storage is fundamental, whether for air conditioning, food preservation, cooling systems, among other applications. This paper presents a numerical analysis by CFD (Computational Fluid Dynamics) about melting of the pure lauric acid PCM (Phase Change Material) into a rectangular cavity with a partial horizontal fin. The numerical model is compound for equations of mass conservation, momentum, energy and phase change. The study consists in validate the employed methodology comparing numerical results with experimental results from the literature. As boundary condition on all cavity walls was used no slip and for the thermal problem was used prescribed temperature on the right vertical wall and fin. The thermal and fluid-dynamic behavior of the lauric acid during the melting was evaluated aiming its application as PCM. Temperature fields, velocity and evolution of liquid fraction over time are presented, showing that the $\mathrm{C}$ parameter that best represents the lauric acid melting is $10^{11}$, and that the natural convection is the most influential mechanism during the melting process.
\end{abstract}

Keywords: PCM; Lauric acid; CFD; Fins.

${ }^{1}$ Universidade do Vale do Rio dos Sinos, Av. Unisinos, 950, Cristo Rei, São Leopoldo, RS, 93020-190, Brasil

${ }^{2}$ Universidade Federal do Rio Grande do Sul, UFRS - Av. Paulo Gama, 110, Farroupilha, Porto Alegre , RS, 90040-060, Brasil.

(*)e-mail: decesaro@unisinos.br

Data de chegada: 07/05/2017 Aceito para publicação: 29/05/2017 


\section{INTRODUÇÃO}

Com a crescente necessidade de racionalizar o uso de energia, o armazenamento de energia térmica tornou-se uma ótima alternativa, visto que permite que uma energia disponível de forma abundante seja utilizada como e quando necessário, gerando um equilíbrio entre oferta e demanda e reduzindo desperdícios. O armazenamento térmico de energia pode ser subdivido em dois grandes sistemas, sendo estes: sistema de armazenamento térmico de energia por calor sensível (SHTES Sensible Heat Thermal Energy Storage) e sistema de armazenamento térmico de energia por calor latente (LHTES - Latente Heat Thermal Energy Storage). Como característica, os SHTES dependem do aumento ou redução de temperatura do material, sendo que a eficácia do sistema é influenciada pelo calor específico e pela massa específica do material, enquanto que os LHTES dependem da capacidade de armazenamento de energia do material em uma condição de temperatura praticamente constante.

Devido ao interesse nos processos de transferência de calor que envolvem mudança de fase, principalmente em função da elevada capacidade de armazenamento térmico, os LHTES se tornaram atrativos em comparação aos SHTES, requerendo menor peso e volume para armazenamento da mesma quantidade de energia. A utilização de materiais de mudança de fase (PCMs - Phase Change Materials) permite avanços nos sistemas térmicos, principalmente no que diz respeito à performance $\mathrm{e}$ rentabilidade, devido às suas características termodinâmicas vantajosas, como grande calor de fusão e armazenamento de quantidades significativas de calor durante o processo de mudança de fase, com pequena variação da temperatura.

Nos últimos anos, diversos estudos sobre o processo de mudança de fase de PCMs foram realizados a fim de verificar o desempenho térmico destes materiais, tanto de forma experimental quanto numérica. Dentre os estudos de forma experimental pode-se citar Tay, Bruno e Belusko (2013), por exemplo, que estudaram a técnica de derretimento dinâmico em um LHTES casco-tubos, verificando que esta técnica melhora a transferência de calor durante a fusão, reduzindo o tempo do processo. Guichard et al. (2015) observaram os efeitos da utilização de PCM em um telhado em clima tropical e úmido, onde concluíram que a inclusão de PCM reduziu a transferência de calor, principalmente por radiação infravermelha, gerando um decréscimo de até $2^{\circ} \mathrm{C}$ na célula de teste. De forma numérica, Sciacovelli, Colella e Verda (2013) estudaram o efeito de nanopartículas no processo de fusão de PCM em um LHTES casco-tubo, onde os resultados demostram um aumento da condutividade térmica, reduzindo em $15 \%$ o tempo de fusão para uma fração volumétrica de $4 \%$ de nanopartíciplas. Tian e Zhao (2011) investigaram o efeito de espumas metálicas na transferência de calor de PCMs, onde foi verificado que a taxa de condução de calor é aumentada significativamente e a convecção natural é suprimida, mas em geral a transferência de calor é melhorada quando espumas metálicas são incorporadas ao PCM.

Segundo Medrano et al. (2009), as aplicações de calor latente com PCM podem ser classificados em dois campos principais: proteção ou inércia térmica e armazenamento de calor. Uma das principais diferenças entre estes dois campos está relacionada à condutividade térmica do PCM, onde em muitos casos de proteção térmica uma baixa condutividade é desejável. Por outro lado, nos sistemas de armazenagem térmica, baixos valores de condutividade térmica são uma desvantagem, visto que a quantidade adequada de energia pode estar disponível, mas o sistema não é capaz de utilizá-la a uma taxa requerida. Devido esta baixa condutividade térmica, buscam-se técnicas para aumento da transferência de calor na maioria das aplicações dos LHTES, das quais podemos citar o encapsulamento, que, segundo Salunkhe e Shembekar (2012), é um processo de revestimento do PCM com um material adequado e que tem por objetivo manter o PCM isolado do material circundante, além de elevar a taxa de transferência de calor. Outra forma bastante usada para aumento da taxa de transferência de calor é a dispersão de micropartículas metálicas, mas segundo Dhaidan et al. (2013) a dispersão de materiais com elevada condutividade térmica não é muito prática, quando comparada à incorporação de estruturas metálicas, uma vez que o material disperso geralmente se aglomera no fundo do recipiente em operações a longo prazo, a não ser que seja utilizada baixa concentração.

Persp. Online: exatas \& eng., Campos dos Goytacazes, 20 (08) 43-55 - 2018

seer.perspectivasonline.com.br 
Conforme Kamkari e Shokouhmand (2014), o uso de aletas metálicas de alta condutividade térmica em TES é uma abordagem simples, confiável e eficaz para aumento da velocidade de fusão. Baby e Balaji (2013) investigaram de forma experimental o comportamento térmico de dois PCMs diferentes em um trocador de calor com aletas tipo pino, variando o número de aletas, onde foi observado que um grande número de aletas uniformemente distribuídas aumenta consideravelmente a transferência de calor. Sciacovelli, Gagliardi e Verda (2015) estudaram de forma numérica a utilização de uma árvore de aletas em forma de Y para melhorar o desempenho térmico de um LHTES casco-tubo, onde foi possível aumentar em $24 \%$ a eficiência do sistema, e além disso observaram que quanto maior o tempo de operação, menor deve ser o ângulo ideal das aletas. Li e Wu (2015) apresentaram um estudo numérico sobre a performance de diversas unidades TES, com geometrias e PCMs variados, onde foi observado que a presença de aletas auxiliou na redução do tempo de solidificação e de fusão. Zauner et al. (2016) realizaram um estudo numérico e experimental sobre a utilização de polímeros de alta densidade como PCMs em uma unidade LHTES do tipo tubo aletado, e seus estudos revelaram que a utilização de polímeros como polietileno (PE), polioximetileno (POM) e poliamida (PA) apresentam resultados promissores apenas mantendo estratégias de controle de fluxo de massa adequadas ou alterando alguns parâmetros de projeto.

Alguns autores focam seus estudos em PCMs de alta temperatura, enquanto outros se aprofundam em PCMs de baixa temperatura (até $120{ }^{\circ} \mathrm{C}$ ) que é o caso do ácido láurico, que foi estudado por Alquaity, Al-Dini e Yilbas (2013) sendo utilizado como nanopartícula para aumento da taxa de transferência de calor da água em microcanais, sendo responsável por aumento na perda de pressão ao longo do comprimento do canal, mas com melhoria na capacidade de armazenamento de calor do fluido. Já Shilei, Neng e Guohui (2006) incorporaram o ácido láurico à materiais de construção a fim de gerar um gesso com armazenamento de energia, onde observaram que este compósito possui boa estabilidade térmica para a temperatura de fusão e variações no calor latente de fusão a longo prazo, podendo ser utilizado para armazenamento de calor latente na conservação de energia de edificações.

Mesmo assim, o ácido láurico não é um material muito estudado, principalmente de forma isolada, então, a fim de determinar novas informações sobre o processo de mudança de fase do ácido láurico e a influência de aletas no processo de transferência de calor, uma abordagem por simulação numérica é utilizada a fim de contemplar fenômenos termodinâmicos que seriam muito difíceis de serem observados e estudados experimentalmente. Este trabalho tem como objetivo investigar numericamente o processo de fusão do PCM ácido láurico em uma unidade de armazenamento retangular com uma aleta parcial horizontal, complementando os resultados experimentais disponíveis na literatura. A geometria do recipiente, as condições iniciais e as condições de contorno das simulações são baseadas no trabalho de Kamkari e Shokouhmand (2014), validando os resultados obtidos. Os mecanismos de transferência de calor envolvidos no processo são analisados por meio de campos de temperatura e de velocidade, juntamente com a evolução da fração líquida ao longo do tempo.

\section{METODOLOGIA}

A unidade de armazenamento possui as seguintes dimensões: $50 \mathrm{~mm}$ de largura, $120 \mathrm{~mm}$ de altura e $120 \mathrm{~mm}$ de profundidade. Ela possui uma aleta parcial horizontal de $25 \mathrm{~mm}$ de comprimento e $4 \mathrm{~mm}$ de espessura no centro da parede direita, conforme representação esquemática mostrada na Fig. 1. A unidade é isolada termicamente e recebe calor apenas através da aleta e da parede da direita. 


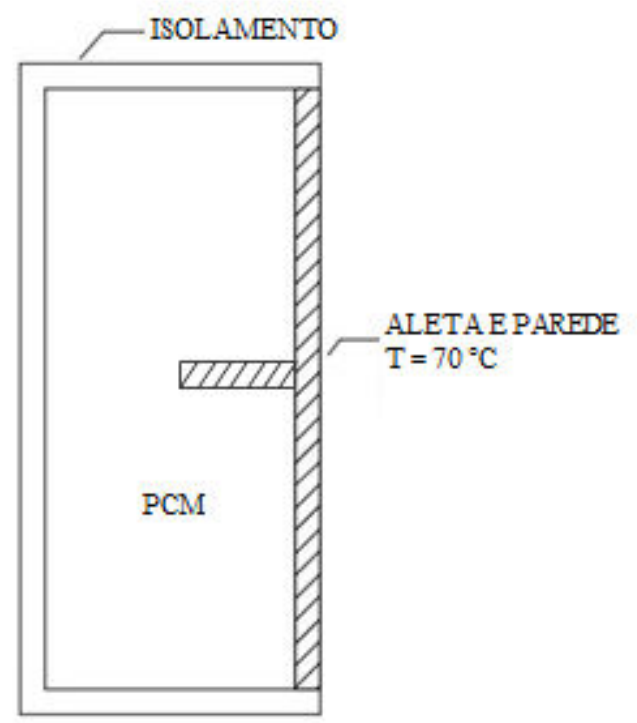

Figura 1: Desenho esquemático

\subsection{Equações governantes}

As equações que compõem o modelo numérico são as equações da conservação da massa (1), da quantidade de movimento (2), da energia (3) e da fração volumétrica (4) (ANSYS FLUENT, 2013).

$\frac{\partial \rho}{\partial t}+\nabla(\rho \vec{V})=0$

$\frac{\partial(\rho \vec{V})}{\partial t}+\nabla(\rho \vec{V} \vec{V})=-\nabla p+\nabla(\mu \nabla \vec{V})+\rho \vec{g}+S$

$\frac{\partial(\rho h)}{\partial t}+\nabla(\rho \overrightarrow{V h})=\nabla(k \nabla T)$

$\frac{\partial\left(\alpha_{n}\right)}{\partial t}+\nabla\left(\alpha_{n} \vec{V}\right)=0$

onde $\rho$ é a massa específica $\left(\mathrm{kg} / \mathrm{m}^{3}\right), t$ é o tempo (s), $\vec{V}$ é o vetor velocidade $(\mathrm{m} / \mathrm{s}), p$ é a pressão $(\mathrm{Pa})$, $\mu$ é a viscosidade dinâmica (Pa.s), $\vec{g}$ é a gravidade $\left(\mathrm{m} / \mathrm{s}^{2}\right), h$ é a entalpia específica $(\mathrm{J} / \mathrm{kg}), k$ é a condutividade térmica (W/m.K), $T$ é a temperatura (K) e $\alpha_{n}$ é a enésima fração volumétrica de PCM na célula computacional.

A entalpia do sistema foi definida como a soma da entalpia sensível $\left(h_{s}\right)$ e a variação da entalpia latente $(\gamma L)$, onde $h_{r e f}$ é a entalpia de referência na temperatura de referência $\left(T_{r e f}\right), c_{p}$ é o calor específico (J/kg.K), $\gamma$ é a fração líquida durante a mudança de fase que ocorre dentro do intervalo de temperatura $T_{s}<T<T_{l}$, e $L$ é a entalpia específica da fusão/solidificação (calor latente do material). As Eq. (5) e (6) para o cálculo dessa variável são definidas por Shmueli, Ziskind e Letan (2010) conforme abaixo:

$h_{s}=h_{r e f}+\int_{T_{r e f}}^{T} c_{p} d T$ 
$\gamma=\left\{\begin{array}{cll}0 & \text { se } & T<T_{s} \\ 1 & \text { se } & T \geq T_{l} \\ \frac{T-T_{s}}{T_{l}-T_{s}} & \text { se } & T_{s} \leq T<T_{l}\end{array}\right.$

O modelo trata a mushy zone, que consiste uma zona de transição líquido-sólido, como uma zona cuja porosidade é igual à fração líquida na célula computacional. Em regiões totalmente solidificadas a porosidade é tida como sendo nula, juntamente com as velocidades de fluxo do material. Devido à esta redução de porosidade em função da fração líquida, o termo $S$ da Eq. (2) é descrito por Assis et al. (2007) conforme a Eq. (7) abaixo:

$\vec{S}=-A_{(\gamma)} \vec{V}$

onde $A_{(\gamma)}$ é a "função da porosidade" definida por Brent, Voller e Reid (1988) e representada pela Eq. (8):

$A_{(\gamma)}=\frac{C(1-\gamma)^{2}}{\left(\gamma^{3}-\varepsilon\right)}$

onde $\varepsilon$ é uma constante de valor 0,001 utilizada para evitar a divisão por zero e $C$ é uma constante que depende da morfologia da mushy zone, segundo Voller e Prakash (1987). A fração líquida $(\gamma)$ para diferentes instantes de tempo é obtida através da Eq. (9), adaptada a partir da equação apresentada por Shmueli, Ziskind e Letan (2010):

$\gamma_{(t)}=\frac{\forall_{(t)}}{\forall_{(t=0)}}$

onde $\forall$ é o volume ocupado pelo PCM na fase líquida $\left(\mathrm{m}^{3}\right)$.

\subsection{Condições iniciais, de contorno e de simulação}

Inicialmente, a unidade está completamente preenchida com PCM sólido, à $25^{\circ} \mathrm{C}$. A parede direita e a aleta são mantidas à $70^{\circ} \mathrm{C}$ e, conforme a fusão evolui, o excesso de PCM líquido dentro da unidade, devido à variação volumétrica, é retirado, a fim de manter a pressão constante. Para simular esta condição, foi criada uma abertura no domínio computacional, posicionada no canto superior direito com $2 \mathrm{~mm}$ de largura. Esta abertura foi modelada como "pressure outlet", com pressão manométrica de $0 \mathrm{~Pa}$ e a mesma temperatura da parede aquecida.

As simulações foram realizadas no software ANSYS Fluent, onde as paredes da unidade foram consideradas na condição de não deslizamento (no-slip). O esquema PRESTO foi utilizado para a equação de correção da pressão, SIMPLE para o acoplamento pressão-velocidade e First Order Upwind para resolver as equações de quantidade de movimento e energia. Utilizou-se um time-step de 0,1 segundos, com limite máximo de 1000 iterações por time-step. Para os critérios de convergência, utilizou-se valores de $10^{-5}$ para as equações de velocidade e de continuidade, e de $10^{-8}$ para a equação de energia.

As malhas computacionais foram criadas no módulo ANSYS-ICEM e são do tipo estruturadas hexaédricas e bidimensionais. Durante o processo de fusão, os maiores gradientes de temperatura e velocidade são encontrados próximos às paredes sólidas, assim, nestas regiões, as malhas receberam um maior refinamento, conforme pode ser observado na Fig. 2. 
Detalhe

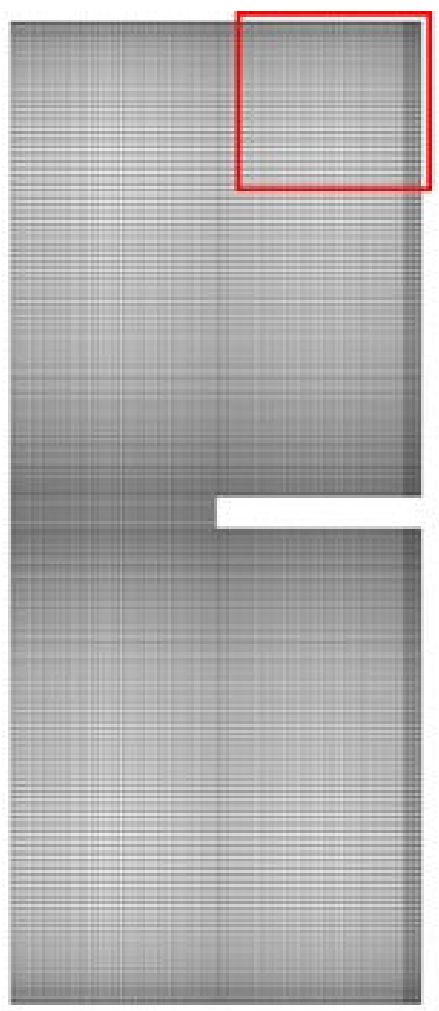

Detalhe
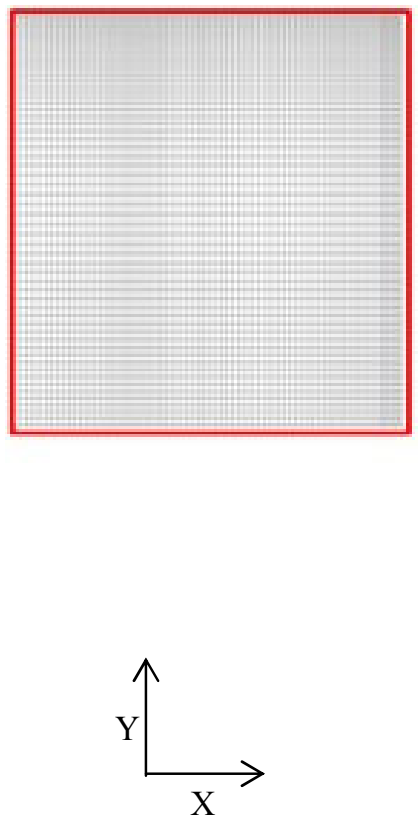

Figura 2: Malha computacional

Para análise de independência de malha, foram testadas três malhas computacionais com: 6872, 28537 e 48640 elementos, denominadas M1 (menos refinada), M2 (intermediária) e M3 (mais refinada), respectivamente.

O PCM utilizado, ácido láurico, também conhecido como ácido dodecanóico $\left(\mathrm{C}_{12} \mathrm{H}_{24} \mathrm{O}_{2}\right)$, é um ácido graxo que está presente em diversas gorduras vegetais, em especial nas que provêm do coco e da semente da palma. Foram utilizados os valores de $k, c_{p}, \rho, L$, das temperaturas de fusão $\left(T_{l}\right)$ e de solidificação $\left(T_{s}\right)$ apresentados por Shokouhmand e Kamkari (2013) e informados nas Tab. 1 e 2.

Tabela 1: Propriedades termofísicas do ácido láurico. Fonte: Shokouhmand e Kamkari (2013)

\begin{tabular}{|c|c|c|c|c|c|c|}
\hline \multicolumn{2}{|c|}{$c_{p}(\mathrm{~kJ} / \mathrm{kg} \mathrm{K})$} & \multirow[t]{2}{*}{$L(\mathrm{~kJ} / \mathrm{kg})$} & \multicolumn{2}{|c|}{$\rho(\mathrm{kg} / \mathrm{m} 3)$} & \multicolumn{2}{|c|}{$T\left({ }^{\circ} \mathbf{C}\right)$} \\
\hline$l$ & $s$ & & $l$ & $S$ & $l$ & $s$ \\
\hline 2,18 & 2,39 & 187,21 & 940 & 885 & 43,5 & 48,2 \\
\hline
\end{tabular}

Considerou-se $\rho$ da fase sólida do ácido láurico como constante. Já os valores de $\rho$ da fase líquida foram obtidos a partir dos estudos de Chuah et al. (2006), sendo: de $870 \mathrm{~kg} / \mathrm{m}^{3}$ para $50^{\circ} \mathrm{C}$, $862 \mathrm{~kg} / \mathrm{m}^{3}$ para $60^{\circ} \mathrm{C}$ e $852 \mathrm{~kg} / \mathrm{m}^{3}$ para $75^{\circ} \mathrm{C}$. Os valores de $\rho, k$ (Tab. 2) e $\mu$ (Tab.3, VALERI E MEIRELLES, 1997), para diferentes temperaturas, foram obtidas por interpolação linear.

Tabela 2: Condutividade térmica do ácido láurico. Fonte: Shokouhmand e Kamkari (2013)

\begin{tabular}{ccccccccc}
\hline $\boldsymbol{T}\left({ }^{\circ} \mathbf{C}\right)$ & $\mathbf{2 0}$ & $\mathbf{3 0}$ & $\mathbf{4 0}$ & $\mathbf{5 0}$ & $\mathbf{5 5}$ & $\mathbf{6 0}$ & $\mathbf{6 5}$ & $\mathbf{7 0}$ \\
\hline$k(\mathrm{~W} / \mathrm{m} \mathrm{K})$ & 0,161 & 0,159 & 0,158 & 0,145 & 0,143 & 0,142 & 0,139 & 0,138 \\
\hline
\end{tabular}

Tabela 3: Viscosidade dinâmica do ácido láurico. Fonte: Valeri e Meirelles (1997)

\begin{tabular}{cccccccc}
\hline $\boldsymbol{T}\left({ }^{\circ} \mathbf{C}\right)$ & $\mathbf{4 8 , 2}$ & $\mathbf{4 9}$ & $\mathbf{6 0}$ & $\mathbf{7 1}$ & $\mathbf{8 2}$ & $\mathbf{9 9}$ & $\mathbf{1 1 0}$ \\
\hline$\mu(\mathrm{kg} / \mathrm{m} \mathrm{s})$ & 0,00760 & 0,00747 & 0,00542 & 0,00428 & 0,00338 & 0,00250 & 0,00210 \\
\hline
\end{tabular}




\section{RESULTADOS E DISCUSSÃO}

A constante $C$, presente na Eq. 8, é responsável pela intensidade de mudança de fase na unidade de tempo, e descreve o processo cinético da zona pastosa. $O$ valor desta constante depende do tipo de PCM utilizado, e normalmente está entre $10^{4}$ e $10^{7}$, mas para o ácido láurico ainda não foram encontrados valores na literatura. Assim, faz-se necessário uma calibração do modelo numérico em termos da constante $C$. A Fig. 3 apresenta resultados da fração líquida pelo tempo, obtidos através do software ANSYS CFD-Post 16.1, para a malha $M 2$ e valores de $C=10^{9}, 10^{10}$ e $10^{11}$, comparando estes com os resultados experimentais de Kamkari e Shokouhmand (2014), onde é possível observar que o valor de $C$ que melhor reproduziu o processo experimental de fusão, mesmo os resultados numéricos sendo muito semelhantes entre si, foi o de $10^{11}$, sendo um resultado diferente da maioria dos valores encontrados na literatura para outros PCMs.

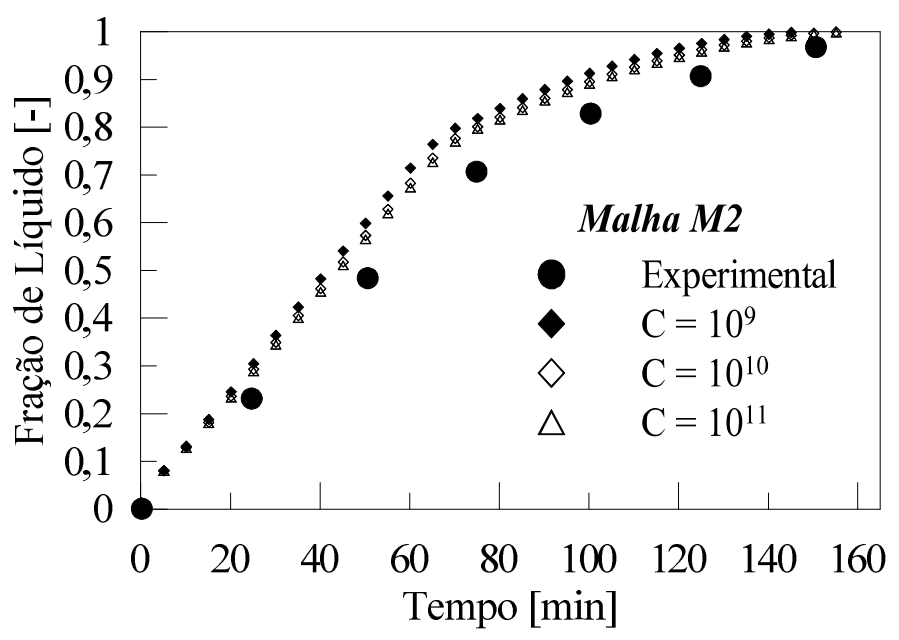

Figura 3: Fração líquida vs. tempo, para $C$ igual a $10^{9}, 10^{10}$ e $10^{11}$ com a malha $M 2$

A fim de analisar a influência do refinamento da malha sobre os resultados apresentados na Fig. 3 (resultados exclusivos com a malha M2), também foram realizadas simulações com as malhas $M 1$ e $M 3$ fixando o valor da constante $C$ em $10^{11}$. A Fig. 4 apresenta resultados de fração líquida ao longo do tempo obtidos de forma experimental e com as três malhas construídas com $C=10^{11}$. Na Fig. 4 é possível observar que de forma quantitativa a malha $M 1$ (menos refinada) é a que apresenta resultados mais próximos ao experimental, ainda mais do que com a malha $M 2$ apresentada na Fig. 3, mas conforme pode ser visto mais adiante (Fig. 5), de forma qualitativa a malha $M 3$ é a que apresenta resultados mais semelhantes aos obtidos experimentalmente. 


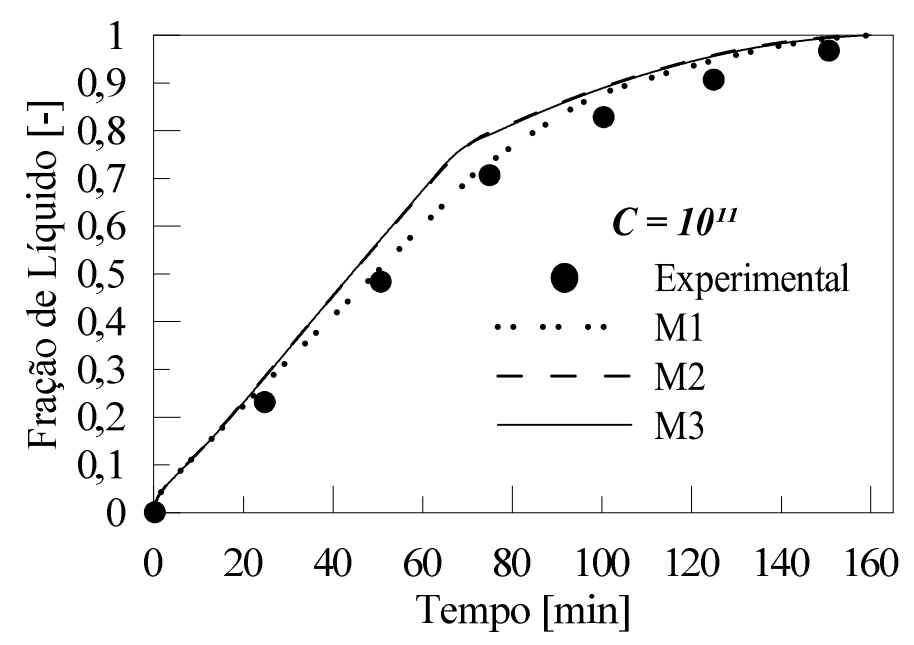

Figura 4: Fração líquida vs. tempo, para malhas $M 1, M 2$ e $M 3$ com $C=10^{11}$

Embora quantitativamente a malha $M 1$ tenha apresentado resultados mais próximos aos experimentais, foi observada uma diferença significativa nos resultados qualitativos, quando comparados com aqueles obtidos experimentalmente por Kamkari e Shokouhmand (2014), como pode ser verificado nas Figs. 5(a-b), que apresentam campos de fração líquida para o tempo de 20 e 50 minutos, obtidos experimentalmente e com as malhas $M 3, M 2$ e $M 1$, respectivamente, com $C=10^{11}$. As cores preto e vermelho representam a fase líquida, enquanto que cinza e azul representam a fase sólida. Comparando-se os diferentes resultados pode-se verificar que os resultados obtidos com as malhas $M 3$ e $M 2$ ficaram praticamente iguais e são os que mais se aproximaram do campo de fração líquida experimental, sendo assim, a fim de reduzir tempos de simulação, a continuidade do trabalho foi realizada com a malha $M 2$.

(a)
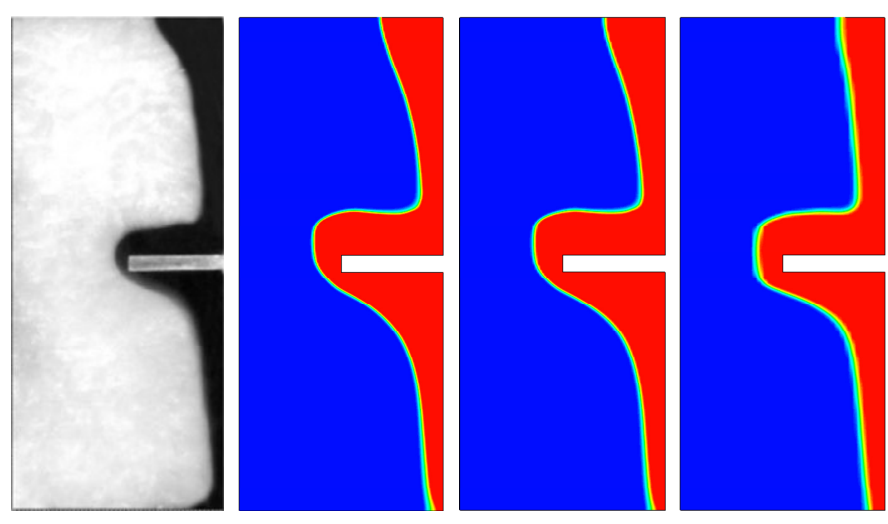

(b)
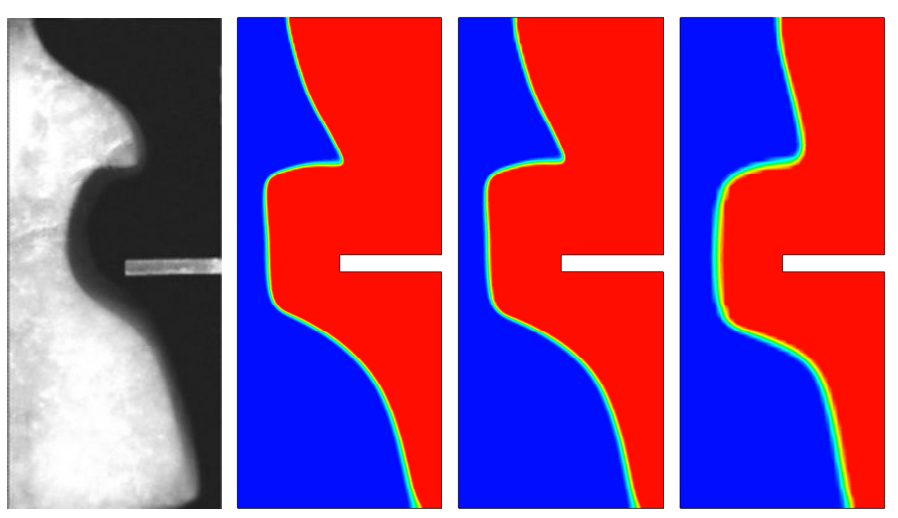

Figura 5: Fração líquida para: (a) $t=20 \min \mathrm{e}$ (b) $t=50 \mathrm{~min}$ 
As Figs. 6(a-b) apresentam campos de velocidade para os tempos de 35 e 60 minutos, respectivamente, com resultados obtidos com a malha $M 2$ e $C=10^{11}$. Pode-se observar que as maiores velocidades do PCM líquido estão localizadas próximas à parede quente e aleta, onde o material é aquecido e sua massa específica reduzida. Esta diferença de massa específica faz com que ocorra um escoamento ascendente do PCM líquido em direção ao topo do recipiente, de encontro à região quente. Escoamentos também foram detectados na interface entre o PCM sólido e líquido, onde o material quente proveniente da região superior do recipiente é resfriado em contato com o sólido, aumentando sua massa específica e escoando em direção à base do recipiente. No detalhe 1 da Fig. 6(b) pode ser observada uma região de recirculação com maiores velocidades, visto que o PCM líquido que está em contato com o PCM sólido tende a descer, encontrando a região da aleta aquecida, aumentando novamente sua temperatura e reduzindo sua massa específica.

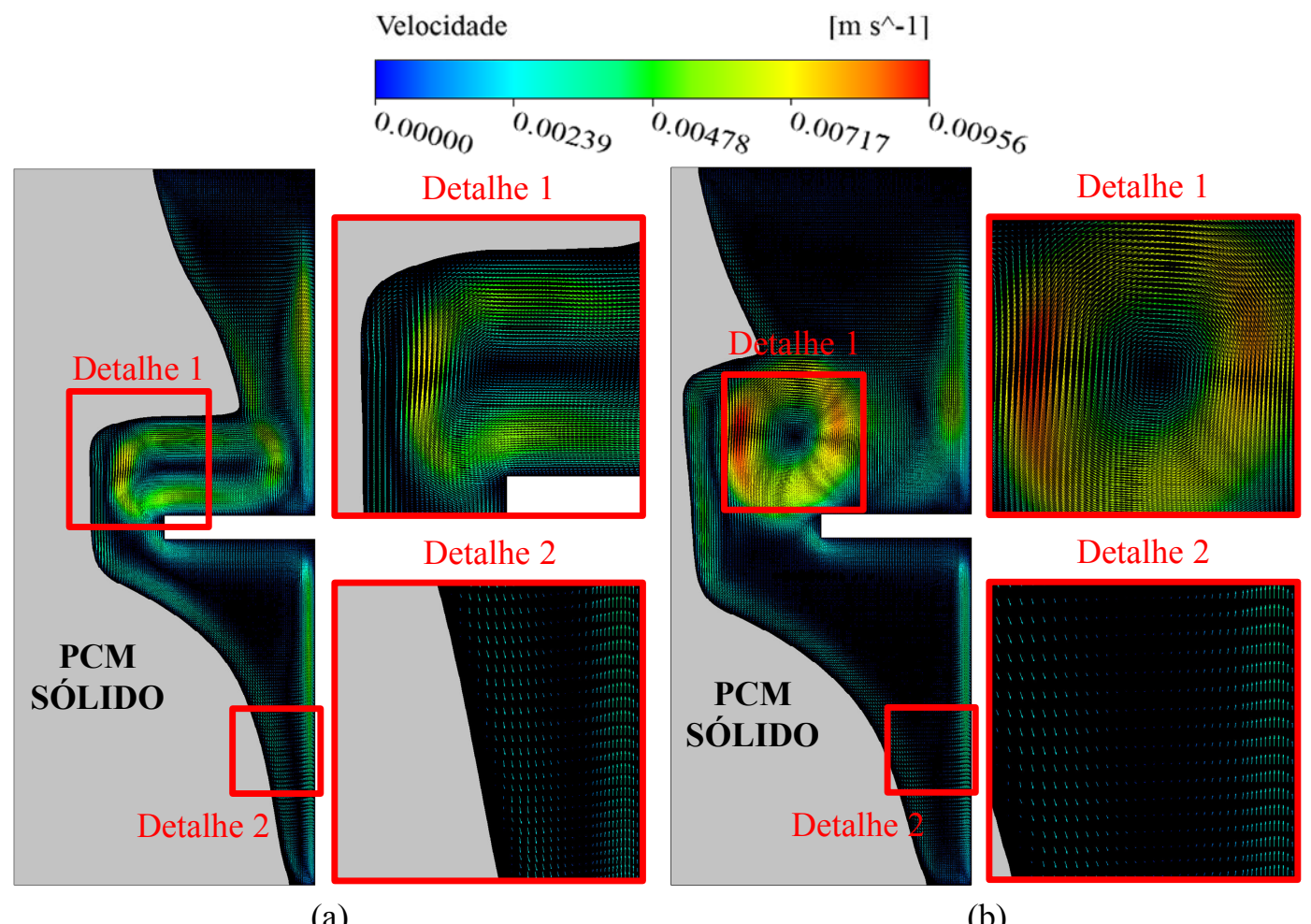

(a)

(b)

Figura 6: Campos de velocidade de PCM líquido em: (a) $t=35 \mathrm{~min}$ e (b) $t=60 \mathrm{~min}$

As Figs. 7(a-d) mostram campos de temperatura para os tempos de 20, 40, 60 e 90 minutos, respectivamente. Com estes campos, pode-se observar que, nos instantes iniciais (Figs. 7(a-b)) a condução é o processo de transferência de calor dominante, enquanto que nos estágios finais (Figs. 7(c-d)), a convecção natural é dominante. Nestas últimas figuras também é possível observar a clara estratificação térmica da fase líquida até a altura corresponde à fase sólida, sendo também visível a camada limite térmica junto à parede vertical direita. $O$ escoamento ascendente faz com que quantidades de massa com diferentes temperaturas sejam deslocadas para uma mesma região, e pela ação do empuxo, essas quantidades de massa tendem a se deslocar para regiões de mesma massa específica, ocorrendo um pequeno retorno de parte do escoamento ascendente. Acima da região sólida a temperatura é praticamente isotérmica na maior temperatura do sistema, o que é uma condição típica de processos de convecção natural. 


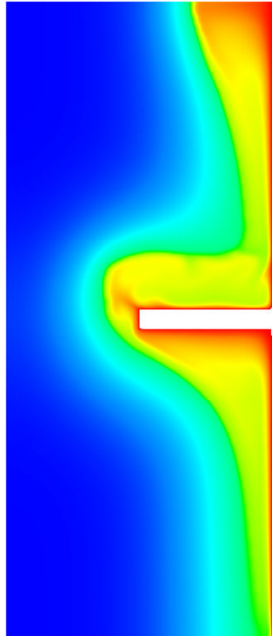

(a)

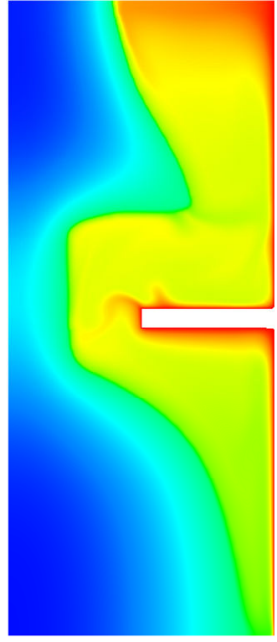

(b)

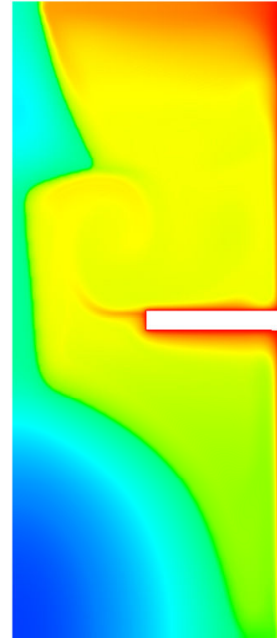

(c)

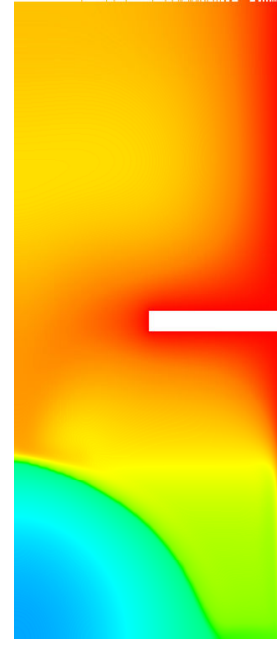

(d)
Temperatura

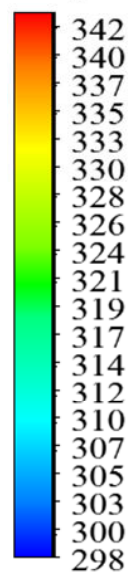

$[\mathrm{K}]$

Figura 7: Campos de temperatura em: (a) $t=20 \mathrm{~min}$, (b) $t=40 \mathrm{~min}$, (c) $t=60 \mathrm{~min}$ e (d) $t=90 \mathrm{~min}$

\section{CONCLUSÕES}

O objetivo deste trabalho era simular o comportamento do processo de fusão do PCM ácido láurico em um recipiente aletado, sendo implementado um modelo matemático e comparando os resultados aos resultados experimentais da literatura. Foi realizada também análise de independência de malha computacional, assim como a influência do parâmetro $C$ do modelo de mudança de fase. A partir dos resultados apresentados, pode-se dizer que:

a) a simulação numérica foi capaz de prever o comportamento da convecção natural e sua atuação sobre a evolução da fração líquida, evidenciada pela maior taxa de derretimento na região superior da fração sólida do PCM;

b) a presença de recirculações na parte superior da cavidade e a aleta influenciam no perfil irregular da fase sólida, visto que estes dois fatores aumentam a transferência de calor. O escoamento de PCM líquido não só aumenta a transferência de calor na região de contato com a mushy zone, como também desgasta a superfície do PCM sólido. Assim, a convecção natural é o mecanismo mais influente no processo de fusão, e seu comportamento deve ser levado em consideração tanto no projeto de recipientes de PCMs quanto escolha do PCM adequado;

c) o parâmetro $C$ da ordem de $10^{11}$ reproduziu de forma satisfatória a fusão do ácido láurico, visto que os resultados quantitativos e qualitativos se mostraram semelhantes aos resultados experimentais.

\section{REFERÊNCIAS}

ALQUAITY, A.B.S., AL-DINI, S.A. \& YILBAS, B.S. Investigation into Thermal Performance of Nanosized Phase Change Material (PCM) in Microchannel Flow. International Journal of Numerical Methods for Heat \& Fluid Flow. Vol. 23, p.233-247, 2013.

ANSYS FLUENT, 2013. Release 14.0. Solver Modeling Guide.

ASSIS, E., KATSMAN, L., ZISKIND, G. \& LETANK, R. Numerical and Experimental Study of Melting in a Spherical Shell. International Journal of Heat and Mass Transfer. Vol. 50, p.1790-1804, 2007.

BABY, R. \& BALAJI, C. Thermal Optimization of PCM Based Pin Fin Heat Sinks: an Experimental Study. Applied Thermal Engineering. Vol. 54, p.65-77, 2013. 
BRENT, A.D., VOLLER, V.R. \& REID, K.J. Enthalpy-porosity Technique for Modeling Convectiondiffusion Phase Change: Application to the Melting of a Pure Metal. Numerical Heat Transfer. Vol. 13, p.297-318, 1988.

CHUAH, T.G., ROZANNA, D., SALMIAH, A., CHOONG, S.Y.T. \& SA'ARI, M. Fatty Acids Used as Phase Change Materials (PCMs) for Thermal Energy Storage in Building Material Applications. JURUTERA. P.8-15, 2006.

DHAIDAN, N.S., KHODADADI, J.M., AL-HATTAB, T.A. \& AL-MASHAT, S.M. Experimental and Numerical Investigation of Melting of NePCM Inside an Annular Container Under a Constant Heat Flux Including the Effect of Eccentricity. International Journal of Heat and Mass Transfer. Vol. 67, p.455-468, 2013.

GUICHARD, S., MIRANVILlE, F., BIGOT, D., MALET-DAMOUR, B. \& BOYER, H. Experimental Investigation on a Complex Roof Incorporating Phase-change Material. Energy and Buildings. Vol. 108, p.36-43, 2015.

KAMKARI, B. \& SHOKOUHMAND, H. Experimental Investigation of Phase Change Material Melting in Rectangular Enclosures with Horizontal Partial Fins. International Journal of Heat and Mass Transfer. Vol. 78, p.839-851, 2014.

LI, Z. \& WU, Z. Analysis of HTFs, PCMs and Fins Effects on the Thermal Performance of Shell-tube Thermal Energy Storage Units. Solar Energy. Vol. 122, p.382-395, 2015.

MEDRANO, M., YILMAZ, M.O., NOGUÉS, M., MARTORELL, I., ROCA, J. \& CABEZA, L.F. Experimental Evaluation of Commercial Heat Exchangers for Use as PCM Thermal Storage Systems. Applied Energy. Vol. 86, p.2047-2055, 2009.

SALUNKHE, P.B. \& SHEMBEKAR, P.S. A Review on Effect of Phase Change Material Encapsulation on the Thermal Performance of a System. Renewable and Sustainable Energy Reviews. Vol. 16, p.5603-5616, 2012.

SCIACOVELLI, A., COLELlA, F. \& VERDA, V. Melting of PCM in a Thermal Energy Storage Unit: Numerical Investigation and Effect of Nanoparticle Enhancement. International Journal of Energy Research. Vol. 37, p.1610-1623, 2013.

SCIACOVELLI, A., GAGLIARDI, F. \& VERDA, V. Maximization of Performance of a PCM Latent Heat Storage System with Innovative Fins. Applied Energy. Vol. 137, p.707-715, 2015.

SHILEI, L., NENG, Z. \& GUOHUI F. Eutectic Mixtures of Capric Acid and Lauric Acid Applied in Building Wallboards for Heat Energy Storage. Energy and Buildings. Vol. 38, p.708-711, 2006.

SHMUELI, H., ZISKIND, G. \& LETAN, R. Melting in a Vertical Cylindrical Tube: Numerical Investigation and Comparison with Experiments. International Journal of Heat and Mass Transfer. Vol. 53, p.4082-4091, 2010.

SHOKOUHMAND, H. \& KAMKARI, B. Experimental Investigation on Melting Heat Transfer Characteristics of Lauric Acid in a Rectangular Thermal Storage Unit. Experimental Thermal and Fluid Science. Vol. 50, p.201-212, 2013.

TAY, N.H.S., BRUNO, F. \& BELUSKO, M. Experimental Investigation of Dynamic Melting in a Tube-in-tank PCM System. Applied Energy. Vol. 104, p.137-148, 2013.

TIAN, Y. \& ZHAO, C.Y. A Numerical Investigation of Heat Transfer in Phase Change Materials (PCMs) Embedded in Porous Metals. Energy. Vol. 36, p.5539-5546, 2011.

VALERI, D. \& MEIRELLES, A.J.A. Viscosities of Fatty Acids, Triglycerides, and their Binary Mixtures. Journal of the American Oil Chemists' Society. Vol. 74, p.1221-1226, 1997.

VOLLER, V.R. \& PRAKASH, C. A Fixed Grid Numerical Modeling Methodology for Convectiondiffusion Mushy Region Phase-change Problems. International Journal of Heat and Mass Transfer. Vol. 30, p.1709-1719, 1987. 
ZAUNER, C., HENGSTBERGER, F., ETZEL, M., LAGER, D., HOFMANN, R. \& WALTER, H. Experimental Characterization and Simulation of a Fin-tube Latent Heat Storage Using High Density Polyethylene as PCM. Applied Energy. Vol. 179, p.237-246, 2016. 